\title{
Invariants and Counterterms in $D=11$ Supergravity
}

\author{
D. Seminara* \\ Laboratoire de Physique Théorique, ${ }^{\dagger}$ École Normale Supérieure \\ F-75231, Paris CEDEX 05, France
}

Abstract: We compute the tree level 4-particle bosonic scattering amplitudes in $\mathrm{D}=11$ supergravity. By construction, they are part of a linearized supersymmetry-, coordinate- and 3-form gauge-invariant. While this on-shell invariant is nonlocal, suitable SUSY-preserving differentiations turn it into a local one with correct dimension to provide a natural lowest (two-loop) order counterterm candidate. Its existence shows explicitly that no symmetries protect this ultimate supergravity from the nonrenormalizability of its lower-dimensional counterparts.

In the post- $\mathrm{D}=10$ superstring era, $\mathrm{D}=11 \mathrm{su}$ pergravity (SUGRA) [1] has again attracted the attention it has always deserved, without however, becoming any easier to handle technically. In particular, supersymmetry (SUSY) invariants are still (absent an appropriate calculus) difficult to verify, let alone construct. Here, we will supply (the linearized bosonic part of) one such invariant. Our work had two motivations beyond its intrinsic interest within the theory. Most directly, we wanted to determine unambiguously whether there exist local invariants that can serve as counterterms at lowest possible, here two-loop, order. This nontrivial exercise has a historical basis in lower-dimensional SUGRAs, where the existence of invariants is easier to decide; there, no miracles occurred: counterterms were always available. They sometimes started at higher order than in pure Einstein gravity (GR) where every loop (except, accidentally, one-loop at $\mathrm{D}=4$ ) is dangerous. [For a recent historical review of divergences in gravities see [2].].] However, given all the properties unique to $\mathrm{D}=11$, and the fact that it is the last frontier - a local QFT that is non-ghost (i.e., has no quadratic curvature terms) and reduces to GR - it is sufficiently important not to give up hope before abandoning

${ }^{*}$ CEE Post-doctoral Fellow under contract FMRX CT 96-0045

†Unité Mixte UMR 8549 associée au Centre de la Recherche Scientifique et à l' École Normale Supérieure.
$\mathrm{D}=11$ SUGRA (and with it all QFTs incorporating GR) too quickly on non-renormalizability grounds. Our second interest is in the M-theoretical direction: any invariants that can be obtained here might provide hints about the wider theory that presumably reduces to $\mathrm{D}=11$ SUGRA as its "zero slope" limit.

The idea underlying our approach is that the set of all $n$-particle (for fixed $n$ ) tree level scattering amplitudes constructed within a perturbative expansion of the action is ipso facto globally SUSY as well as linearized coordinate- and 3form gauge invariant. Thus, because linearized SUSY does not mix different powers of fields, the 4-point amplitudes of interest to us, taken together, form an invariant. Also, within this lowest order framework, the bosonic amplitudes are independent of fermions: virtual ones cannot contribute at tree level. The above statements together considerably lighten our task, which will be to compute "just" the parts involving the gravitational and form bosonic excitations. The amplitudes involving fermions are not necessarily more complicated, merely less relevant to our immediate goal of reproducing terms about which the appropriate divergence computations exist; indeed, we hope to return to them [3i]. However, in order to use the scattering amplitudes for counterterm purposes, it will first be necessary to strip them of the nonlocality associated with exchange of the virtual graviton and form particles 
("formions") without compromising their invariances. Actually, the task here will be not only to remove nonlocality but to add sufficient further powers of momentum to provide an on-shell invariant of correct dimension that is an acceptable (and indeed first possible) perturbative counterterm candidate. In this way, we will make contact with the conclusive 2-loop results of [i] was possible to exhibit the infinity of a local 4graviton term, one that is precisely a component of our invariant. An earlier version of our results

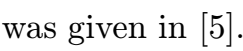

The basis for our computations is the full $\mathrm{D}=11$ SUGRA action [1] $[1,1]$, expanded to quartic order in its two bosons, namely the graviton and the formion, with three-form potential $A_{\mu \nu \rho}$. The field strength $F_{\mu \nu \alpha \beta} \equiv 4 \partial_{[\mu} A_{\nu \alpha \beta]}$ is invariant under the gauge transformations $\delta A_{\nu \nu \alpha}=\partial_{[\mu} \xi_{\nu \alpha]}$, square brackets denoting total (normalized) antisymmetrization. The bosonic truncation of the Lagrangian is

$$
\begin{aligned}
& \mathcal{L}_{B}=-\frac{\sqrt{-g}}{4 \kappa^{2}} R-\frac{\sqrt{-g}}{48} F_{\mu \nu \rho \sigma} F^{\mu \nu \rho \sigma}+ \\
& \frac{2 \kappa}{144^{2}} \epsilon^{1 . .11} A_{1-3} F_{4-7} F_{8-11} .
\end{aligned}
$$

The metric signature in eq. (ii i is mostly minus, the Ricci tensor is defined by $R_{\alpha \beta} \sim+\partial_{\lambda} \Gamma_{\alpha \beta}^{\lambda}$, and the Levi-Civita symbol obeys $\epsilon^{0 \ldots 10}=-1$. The gravitational constant $\kappa$, with dimension $[L]^{\frac{9}{2}}$ also appears explicitly in the topological $(P, T)-$ conserving metric-independent Chern-Simon (CS) part of $\left(\begin{array}{l}\overline{1} \\ 1\end{array}\right)$.

The propagators and vertices required for our computations are obtained by expanding in powers of $\kappa$, with $g_{\mu \nu} \equiv \eta_{\mu \nu}+\kappa h_{\mu \nu}$ and keeping all contributions through order $\kappa^{2}$. The propagators, from the quadratic part of the action, are well known. In harmonic (de Donder) and Feynman gauges for gravity and the 3 -form respectively,

$$
D^{\mu \nu ; \alpha \beta}(h) \equiv G^{\mu \nu ; \alpha \beta} D_{F}
$$

with $G^{\mu \nu ; \alpha \beta}=\frac{1}{2}\left(\eta_{\alpha \mu} \eta_{\beta \nu}+\eta_{\alpha \nu} \eta_{\beta \mu}-\frac{2}{9} \eta_{\alpha \beta} \eta_{\mu \nu}\right)$ and

$$
D^{\mu \nu \rho ; \alpha \beta \sigma}(A)=\frac{1}{2} \delta_{\alpha \beta \sigma}^{\mu \nu \rho} D_{F} ;
$$

$D_{F}$ is the scalar Feynman propagator and $\delta_{\alpha \beta \sigma}^{\mu \nu \rho}$ is totally antisymmetric in each triplet of indices.
There are three cubic vertices:

(a) Three gravitons $\left(h^{3}\right)$. Explicit use of this cumbersome vertex can be avoided in dealing with the four-graviton amplitudes, but not in computing the graviton-form "Compton" scattering. To minimize the complications, we write the vertex already contracted with two on-shell polarization tensors, since we will never need fewer contractions:

$$
\begin{aligned}
& 2 V_{\mu \nu ; \alpha \beta ; \rho \sigma}\left(k^{3}, k^{1}, k^{2}\right) \epsilon_{1}^{\alpha} \epsilon_{1}^{\beta} \epsilon_{2}^{\rho} \epsilon_{2}^{\sigma}= \\
& \left(\epsilon_{1} \cdot k_{2}\right)^{2} \epsilon_{2}^{\mu} \epsilon_{2}^{\nu}-\left(\epsilon_{1} \cdot k_{2}\right)\left(\epsilon_{2} \cdot k_{1}\right)\left(\epsilon_{1}^{\mu} \epsilon_{2}^{\nu}+\epsilon_{2}^{\mu} \epsilon_{1}^{\nu}\right) \\
& +\left(\epsilon_{2} \cdot k_{1}\right)^{2} \epsilon_{1}^{\mu} \epsilon_{1}^{\nu}+\left(\epsilon_{1} \cdot \epsilon_{2}\right)\left[\left(\epsilon_{1} \cdot k_{2}\right)\left(\epsilon_{2} \cdot k_{1}\right) \eta^{\mu \nu}\right. \\
& -\left(\epsilon_{1} \cdot k_{2}\right) \epsilon_{2}^{\mu} k_{2}^{\nu}-\left(\epsilon_{1} \cdot k_{2}\right) \epsilon_{2}^{\nu} k_{2}^{\mu}-\left(\epsilon_{2} \cdot k_{1}\right) \epsilon_{1}^{\mu} k_{1}^{\nu} \\
& \left.+\left(\epsilon_{1}^{\mu} \epsilon_{2}^{\nu}+\epsilon_{2}^{\mu} \epsilon_{1}^{\nu}\right) k_{1} \cdot k_{2}-\left(\epsilon_{2} \cdot k_{1}\right) \epsilon_{1}^{\nu} k_{1}^{\mu}\right] \\
& +\left(\epsilon_{1} \cdot \epsilon_{2}\right)^{2}\left[k_{1}^{\mu} k_{1}^{\nu}+1 / 2 k_{1}^{\mu} k_{2}^{\nu}+1 / 2 k_{1}^{\nu} k_{2}^{\mu}+k_{2}^{\mu} k_{2}^{\nu}\right. \\
& \left.-3 / 2 \eta^{\mu \nu} k_{1} \cdot k_{2}\right]
\end{aligned}
$$

Here and throughout the polarization tensor $\epsilon^{\alpha \beta}$ of a graviton is represented as the product $\epsilon_{i}^{\alpha} \epsilon_{i}^{\beta}$ of two polarization vectors.

(b) Graviton-form $(h F F)$ : this is the usual coupling between the metric and the form's stress tensor. In coordinate space,

$$
\begin{aligned}
& V_{3}^{g F F}=\kappa T_{\mu \nu} h^{\mu \nu}= \\
& =\kappa h^{\mu \nu}\left(F_{\mu \alpha \beta \rho} F_{\nu}{ }^{\alpha \beta \rho}-\frac{1}{8} \eta_{\mu \nu} F_{\alpha \beta \rho \sigma} F^{\alpha \beta \rho \sigma}\right)(5) \\
& =\kappa A_{\alpha \beta \rho} \partial_{\mu}\left(h^{\nu[\mu} F_{\nu}{ }^{\alpha \beta \rho]}-\frac{h}{2} F^{\mu \alpha \beta \rho}\right) .
\end{aligned}
$$

Expressions (5,6) differ (onshell and for harmonic gauge) by an integration by parts: the former is the more suitable in the analysis of pure form scattering, the latter for graviton-form Compton scattering. Note that both expressions simplify if we choose a gauge where $h_{\mu \nu}$ is traceless.

(c) Three formions $(A F F)$ : Due entirely to the (metric independent) CS term in $\left(\overline{1} \overline{1}_{1}^{\prime}\right)$, it is usefully written as $V_{3}^{F}=A_{\mu \nu \alpha} C_{F}^{\mu \nu \alpha}$ with

$$
C_{F}^{\rho \sigma \tau} \equiv \frac{2}{(12)^{4}} \epsilon^{\rho \sigma \tau 1 . .8} F_{1 . .4} F_{5 . .8}
$$

This vertex will produce a non-gravitational contribution to 4-formion scattering and will also be responsible for an unusual, $F^{3} R$ "bremsstrahlung", amplitude.

Finally, to achieve gauge invariance, we must also include the effects of two four-point contact 
$\left(\kappa^{2}\right)$ vertices. The first is the local 4-graviton vertex; it will not be written out here, but is needed for the 4-graviton amplitude calculation. The second is the $h h F F$ vertex from expanding the $F^{2}$ kinetic term in (1); it is necessary to insure gauge invariance in the graviton-formion Compton process. Its form, in a gauge where the graviton is traceless, is

$$
\begin{aligned}
& V^{h h F F}=\frac{1}{48} \delta^{2} \int \sqrt{-g} F^{2} /\left.\delta g_{\mu \nu} \delta g_{\alpha \beta}\right|_{g=\eta} h_{\mu \nu} h_{\alpha \beta}= \\
& -\frac{\kappa^{2}}{48}\left[4 h^{\mu}{ }_{\lambda} h^{\nu \lambda} F_{\mu \alpha \beta \rho} F_{\nu}{ }^{\alpha \beta \rho}+\right. \\
& \left.3 h_{\mu \nu} h^{\mu \nu} F_{\alpha \beta \rho \sigma} F^{\alpha \beta \rho \sigma}-24 h_{\mu \nu} h_{\alpha \beta} F_{\rho \sigma}^{\mu \alpha} F^{\nu \beta \rho \sigma}\right]
\end{aligned}
$$

In the following we outline the explicit computation of (the bosonic part of) the SUSY invariant amplitude and then construct the corresponding local invariants. Before entering into details, some general remarks are in order. In momentum space, the non-locality in each scattering amplitude (due to the intermediate denominator of the exchanged particle) is represented by a sum of simple poles, in each of the Mandelstam variables $(s, t, u)$, corresponding to the three different possible channels in four-particle scattering; this nonlocality is easily neutralized by multiplying the final result by the symmetric polynomial stu. Since multiplication in momentum space corresponds to differentiation in coordinate space, it becomes necessary to understand how these additional derivatives are to be spread. Suppose that we can write the amplitude in the generic "current-current" single pole form, as follows

$$
\begin{aligned}
& M=\phi_{1}^{a}\left(k_{1}\right) \phi_{2}^{b}\left(k_{2}\right) V_{a b m}\left(k_{1}, k_{2}\right) \frac{G^{m n}}{s} \\
& W_{m c d}\left(k_{3}, k_{4}\right) \phi_{3}^{c}\left(k_{3}\right) \phi_{4}^{d}\left(k_{4}\right)+(s t u) \text { perm.. }(8)
\end{aligned}
$$

Then, by multiplying by stu and using the identity

$$
\begin{aligned}
& t u=\frac{1}{2}\left(\eta^{\mu \nu} \eta^{\alpha \beta}-\eta^{\mu \alpha} \eta^{\nu \beta}-\eta^{\nu \alpha} \eta^{\mu \beta}\right) k_{\mu}^{1} k_{\nu}^{2} k_{\alpha}^{3} k_{\beta}^{4} \\
& \equiv-\frac{1}{2} K^{\mu \nu ; \alpha \beta} k_{\mu}^{1} k_{\nu}^{2} k_{\alpha}^{3} k_{\beta}^{4}
\end{aligned}
$$

and its permutations, we can write

$$
\begin{gathered}
M=k_{\mu}^{1} \phi_{1}^{a}\left(k_{1}\right) k_{\nu}^{2} \phi_{2}^{b}\left(k_{2}\right) V_{a b m}\left(k_{1}, k_{2}\right) K^{\mu \nu ; \alpha \beta} G^{m n} \\
W_{m c d}\left(k_{3}, k_{4}\right) k_{\alpha}^{3} \phi_{3}^{c}\left(k_{3}\right) k_{\beta}^{4} \phi_{4}^{d}\left(k_{4}\right)+(s t u) \text { perm.(10) }
\end{gathered}
$$

In other words, if we Fourier-transform back to coordinate space, the net effect of this procedure is to remove the pole and to add a derivative to each of the four external fields. These new derivatives are to be contracted according to the $K^{\alpha \beta ; \mu \nu}$ matrix defined in $\left(\overline{\bar{p}^{\prime}}\right)$. If the amplitude is already expressed as a product of gauge invariant currents, this procedure produces an invariant that is the product of two new dressed gaugeinvariant currents. In the case of gravitationally induced matter interactions, these currents behave like counterparts of the Bel-Robinson (BR) tensors [i, $[\overline{6}]$.

The above "dressing" procedure leaves unaltered an amplitude's transformation under global symmetries, such as the linearized supersymmetry of interest: We are just multiplying an invariant by a numerical factor, the derivatives. While there will be some exceptions in detail to application the above remarks, the final local results achieved will be correct, i.e., we have a constructive procedure for transforming the guaranteed symmetry-preserving but nonlocal amplitudes into equally invariant (on-shell) local terms.

We start with the 4-graviton amplitude $M_{4}^{g}$. The graviton exchange contributions stem from (a) contracting two $V_{g}^{3}$ vertices (4) in all three $(s, t, u)$ channels via an intermediate graviton propagator (2), which provides a single denominator and (b) the local 4-point vertex $V_{4}^{g}$. The resulting $M_{4}^{g}(h)$ will be a non-local quartic polynomial in the Riemann (Weyl, on linear shell) tensor, whose non-locality is removable by stumultiplication. In $\mathrm{D}=4$, most of the calculation can be avoided because a straightforward implementation of supersymmetry allows one to fix the amplitude completely up to normalization: There are only two independent local scalar quartics in the Weyl tensor and its dual, ${ }^{*} R$ : the squares of Euler $\left(E_{4} \equiv{ }^{*} R^{*} R\right)$ and Pontryagin $\left(P_{4} \equiv{ }^{*} R R\right)$ densities. Their relative coefficient can be determined by exploiting the special property that ensures the supersymmetrizability of the Einstein action, namely that it is, at tree level, maximally helicity conserving [i]7]. This constrains the amplitude to be proportional to the combination $\left(E_{4}-P_{4}\right)\left(E_{4}+P_{4}\right)$. Remarkably, this invariant is also, owing to identities peculiar to $\mathrm{D}=4$, expressible as the square of the 
(unique) BR tensor $B_{\mu \nu \alpha \beta}=\left(R R+{ }^{*} R^{*} R\right)_{\mu \nu \alpha \beta}$. Unfortunately, $D=4$ is highly degenerate. In generic dimension, which in this context means $D \geq 8$, the number of invariants quartic in the Weyl tensor is seven and the only condition given by the above constraint is obviously not enough to fix the relative coefficients. Nevertheless it is still sufficient to determine the amplitude completely by considering configurations where the helicities of the gravitons belong to the subspace defined by their four momenta.

A further step can be taken using a very different property, which is not manifest from the GR action, having a string origin: The 4-graviton tree amplitude is proportional to the square of "bleached" 4-gluon tree amplitudes, upon representing the graviton polarization tensor as the product of two vectors; this is implied by the field theory limit of the KLT [i] ristions. This additional information in fact, determines the amplitude completely, because maximal helicity conservation fixes the (uncolored) 4-gluon amplitude (since there are only two independent $F^{4}$ invariants in any $D$ ) and consequently the gravity amplitude, which is its square. The conclusion that the form of $M_{4}^{g}$ is

$$
\begin{aligned}
& M_{4}^{g} \propto(s t u)^{-1} t_{8}^{\mu_{1} \cdots \mu_{8}} t_{8}^{\nu_{1} \cdots \nu_{8}} \times \\
& R_{\mu_{1} \mu_{2} \nu_{1} \nu_{2}} R_{\mu_{3} \mu_{4} \nu_{3} \nu_{4}} R_{\mu_{5} \mu_{6} \nu_{5} \nu_{6}} R_{\mu_{7} \mu_{8} \nu_{7} \nu_{8}}
\end{aligned}
$$

follows from the gluon "square root" (in this context, $F_{\mu \nu}$ stands for the gluon field strength)

$$
\begin{aligned}
& M_{4}^{\text {gluon }} \propto t_{8}^{\mu_{1} \cdots \mu_{8}} F_{\mu_{1} \mu_{2}} \cdots F_{\mu_{7} \mu_{8}}= \\
& \left(F_{\mu \nu} F^{\mu \nu}\right)^{2}-4 F^{\mu_{1} \mu_{2}} F_{\mu_{2} \mu_{3}} F^{\mu_{3} \mu_{4}} F_{\mu_{4} \mu_{1}} .
\end{aligned}
$$

Alternatively one can follow the explicit calculational steps spelled out at the beginning. The algebra involved is quite cumbersome, and benefits from a program for algebraic manipulation. This analysis should obviously lead to the same result and indeed it does. Still, it must be performed, at least for a particular set of helicities, in order to obtain the correct normalization of the amplitudes. For example, by choosing a configuration such that $\epsilon_{i} \cdot k_{j}=0$ for all $i$ and $j$, one finds that the overall coefficient of $\left(\overline{1}_{1} \overline{1}_{i}^{\prime}\right)$ is fixed to be $1 / 4$. The final result $\left(\overline{1}_{1} \overline{1}_{1}^{\prime}\right)$ possesses the same tensorial structure as the familiar superstring zero-slope limit correction to $\mathrm{D}=10 \mathrm{~N}=2$ supergravity, where the $t_{8}^{\mu \cdots \mu_{8}}$ symbol originates from the $\mathrm{D}=8$ transverse subspace $[\overline{9} \mid$, as has also been noted in [1] [1] which carried out the direct 4-graviton calculations as well. This reflects the fact that maximal supersymmetry implies a unique $R^{4}$ in all dimensions. If we assume only $1 / 2$ of the maximal supersymmetry in generic $D$ we find that there is room for two invariants, as can be seen by looking, e.g. at the effective action of the heterotic superstring where the analog of $\left(\overline{1}_{1} \overline{1}_{1}^{\prime}\right)$ is accompanied by another $R^{4}$ term.

At this point it is quite easy to write down a combination of local $R^{4}$ that represents $\left(1 \overline{1} \overline{1}_{1}^{\prime}\right)$. The Lagrangian is

$$
\begin{aligned}
& L_{4}^{g}=\frac{1}{4} R^{\alpha \beta \rho \sigma} R_{\alpha \beta}{ }^{\lambda \mu} R_{\rho \lambda}{ }^{\nu \omega} R_{\sigma \mu \nu \omega},- \\
& R^{\alpha \beta \rho \sigma} R_{\alpha}^{\lambda{ }^{\mu}{ }^{\mu}} R_{\lambda{ }^{\nu}{ }^{\nu}{ }^{\omega}} R_{\mu \nu \sigma \omega} .
\end{aligned}
$$

where we have dropped a term proportional to the 8-dimensional Euler density $\left(\epsilon_{8} \epsilon_{8} R^{4}\right)$ that, being a total divergence to leading order, does not affect the amplitude. In many respects, the form $\left(\overline{1}_{1} \overline{1}_{i}\right)$ for the contribution coming from the 4-graviton amplitude, is a perfectly physical one. However, one might wonder whether there is a formulation of the above Lagrangian in terms of currents that encompasses both gravity and matter in a unified way as in fact occurs in e.g. $N=2, D=4$ supergravity [1 $\left.{ }_{1}^{1}\right]$. This might also lead to some understanding of higher spin SUSY multiplets. One may rewrite $L_{4}^{g}$ in various ways involving the BR current $B_{\mu \nu \alpha \beta}$,

$$
\begin{aligned}
& {\left[R_{\mu \rho \alpha \sigma} R_{\nu \beta}^{\rho}{ }^{\sigma}+(\nu \mu)\right]-\frac{1}{2} g_{\mu \nu} R_{\alpha \rho \sigma \tau} R_{\beta}{ }^{\rho \sigma}(14)} \\
& -\frac{1}{2} g_{\alpha \beta} R_{\mu \rho \sigma \tau} R_{\nu}^{\rho \sigma \tau}+\frac{1}{8} g_{\mu \nu} g_{\alpha \beta} R_{\lambda \rho \sigma \tau} R^{\lambda \rho \sigma \tau}
\end{aligned}
$$

and the closed 4 -form $P_{\alpha \beta \mu \nu}=1 / 4 R_{[\mu \nu}^{a b} R_{\alpha \beta] a b}$. For example if we choose the BR tensor we can write

$$
\begin{aligned}
& L_{4}^{g}=48 \kappa^{2}\left[2 B_{\mu \nu \alpha \beta} B^{\mu \alpha \nu \beta}-B_{\mu \nu \alpha \beta} B^{\mu \nu \alpha \beta}+(15)\right. \\
& \left.6 B_{\mu \rho \alpha}{ }^{\rho} B_{\sigma}^{\mu \sigma \alpha}-\frac{15}{49}\left(B_{\mu \nu}^{\mu \nu}\right)^{2}+P_{\mu \nu \alpha \beta} P^{\mu \nu \alpha \beta}\right] .
\end{aligned}
$$

Due to the larger number of allowed invariants and of helicities in $\mathrm{D}=11$, this representation does not seems to share the elegance and power of the four dimensional one. Still, it is remarkably compact. 
We turn now to pure formion scattering. This amplitude is quite simple to investigate because it must be manifestly (form) gauge invariant: the three-form potential $A$ only appears in the operative vertices $(5,7)$ through its curvature $F$; the relevant currents are in fact the $\mathrm{CS} C_{\mu \nu \alpha}^{F}$ and the stress tensor $T_{\mu \nu}^{F}$. The interactions are mediated respectively by the formion and the graviton. Therefore the amplitude is already organized in terms of gauge invariant currents; indeed we have, in terms of $T_{F}, C_{F}$ of $(5,7)$,

$$
\begin{gathered}
M_{F 4}^{\text {grav-med. }}=\left(\frac{\kappa}{6}\right)^{2} \times \\
\left(T_{F}^{\alpha \beta}\left(k_{1}, k_{2}\right) \frac{G_{\alpha \beta ; \mu \nu}}{s} T_{F}^{\mu \nu}\left(k_{3}, k_{4}\right)+\text { perm. }\right) .
\end{gathered}
$$

and

$$
\begin{aligned}
& M_{F 4}^{\text {form-med. }}=-\left(\frac{\kappa}{48}\right)^{2} \times \\
& \left(C^{F \alpha \beta \rho}\left(k_{1}, k_{2}\right) \frac{1}{3 s} C_{\alpha \beta \rho}^{F}\left(k_{3}, k_{4}\right)+\text { perm. }\right),
\end{aligned}
$$

where "perm" stands for permutation of the four external particles. The sum of (16) and (17) agrees with a recent calculation of formion scattering from a quite different starting-point [12]']. We must now multiply our total $M_{F 4}$ by stu and see how the derivatives spread. Using the simple rule stated above, we recognize immediately that there is an economical way of organizing $L_{4}^{F}$ in terms of matter BR and of the corresponding $C^{F}$ extensions. In fact if we define

$$
\begin{aligned}
& B_{\mu \nu \alpha \beta}^{F} \equiv \partial_{\alpha} F_{\mu} \partial_{\beta} F_{\nu}+\partial_{\beta} F_{\mu} \partial_{\alpha} F_{\nu}-\frac{1}{4} \eta_{\mu \nu} \partial_{\alpha} F \partial_{\beta} F, \\
& C_{\rho \sigma \tau ; \alpha \beta}^{F} \equiv \frac{1}{(24)^{2}} \epsilon_{\rho \sigma \tau \mu_{1} \cdots \mu_{8}} \partial_{\alpha} F^{\mu_{1} \cdots \mu_{4}} \partial_{\beta} F^{\mu_{5} \cdots \mu_{8}} .
\end{aligned}
$$

with $\partial^{\mu} B_{\mu \nu \alpha \beta}^{F}=0$ and $\partial^{\rho} C_{\rho \sigma \tau ; \alpha \beta}^{F}=0$ and where implicit indices are summed in the obvious way, then

$$
\begin{aligned}
& L_{4}^{F}=\frac{\kappa^{2}}{36} B_{\mu \nu \alpha \beta}^{F} B_{\mu_{1} \nu_{1} \alpha_{1} \beta_{1}}^{F} G^{\mu \mu_{1} ; \nu_{1} \nu} K^{\alpha \alpha_{1} ; \beta_{1} \beta}- \\
& \frac{\kappa^{2}}{12} C_{\mu \nu \rho ; \alpha \beta}^{F} C_{\alpha_{1} \beta_{1}}^{F \mu \nu \rho} K^{\alpha \alpha_{1} ; \beta_{1} \beta} .
\end{aligned}
$$

Reflecting its simple "current-current" origin, the pure matter sector has a natural (if perhaps not unique) expression in terms of currents. There is also a basis of scalars quartic in $F$; we have not used it here, but it is tabulated in the Appendix.
Here there is just one diagram, namely the emission of a graviton described by the stress tensor vertex $(5,6)$, from any of the 3 formion lines emanating out of the CS vertex (7). The analysis of this amplitude follows the lines of the previous one. While it is not manifestly (gravitationally) gauge-invariant, its invariance can be verified using the following local $\mathrm{D}=11$ identity

$$
d(A \wedge F \wedge F) \equiv F_{\alpha \mu_{1} \ldots} F_{\ldots} F_{\ldots \mu_{11}} \epsilon^{\mu_{1} \cdots \mu_{11}}=0 .
$$

This identity enables us to write the amplitude schematically in the form

$M_{4}^{h F^{3}}=h^{\mu \nu}\left(F_{\mu \alpha \beta \rho} G_{\nu}{ }^{\alpha \beta \rho}-\frac{1}{8} \eta_{\mu \nu} G_{\alpha \beta \rho \sigma} F^{\alpha \beta \rho \sigma}\right)$

plus the obvious permutations that restores the $s u$ symmetry of the process; $G_{\mu \nu \alpha \beta}$ is the effective field strength (obeying the $F$ equation of motion) constructed out of the "connection" defined by $(\triangle)^{-1} C_{F}^{\alpha \beta \rho}$ with the $C_{F}$ of (7). Then the gauge invariance of the amplitude is equivalent to the conservation of the "energy momentum tensor" effectively defined in $\left(20_{1}^{i}\right)$. Next we again multiply derivatives according to the rule given previously. Turning the $h_{\mu \nu}$ in (20 $\left.\overline{0}_{1}^{\prime}\right)$ into a Riemann tensor takes some patience and a certain number of integration by parts, however. The final result is

$$
\begin{aligned}
L_{4}^{F F F g}= & (s t u) M_{4}^{F F F g}= \\
& -\frac{\kappa^{2}}{3} C_{\mu \nu \rho ; \alpha \beta}^{F} C_{\alpha_{1} \beta_{1}}^{R F \mu \nu \rho} K^{\alpha \alpha_{1} ; \beta_{1} \beta},
\end{aligned}
$$

where $C_{\mu \nu \rho ; \alpha \beta}^{R F}$ is given by

$$
4 \partial_{\lambda}\left(R_{(\alpha \beta)}^{\sigma[\lambda}{ }^{[\lambda} F_{\sigma}^{\mu \nu \rho]}\right)-\frac{2}{3} R_{(\alpha}^{\left.\sigma{ }^{\lambda} \beta\right)} \partial_{\lambda} F_{\sigma}{ }^{\mu \nu \rho} .
$$

To prove this result, we used the following generalization of the identity $\left(\overline{1}_{\mathbf{L}} \overline{9}_{-}^{\prime}\right)$

$$
\partial_{\alpha} \partial_{\beta} F_{\alpha \mu_{1} \mu_{2} \mu_{3}} \partial^{\alpha} F_{\mu_{4} \cdots \mu_{7}} \partial^{\beta} F_{\mu_{8} \cdots \mu_{11}} \epsilon^{\mu_{1} \cdots \mu_{11}} \equiv 0 .
$$

While it is clear that a " $C^{R F}$ current" must exist since $C^{F}$ factorizes the amplitude, [?] is not unique and we claim no special significance for it.

The most complicated amplitude is that for graviton-formion scattering. It involves two classes of diagrams. The first consists of the $T_{\mu \nu}^{F}$ stress 
tensor turning into two gravitons via graviton exchange between the vertices $h^{\mu \nu} T_{\mu \nu}^{F}$ of (6) and the $h \partial h \partial h$ of (4) along with the mixed quartic contact term required to preserve gauge invariance. The second set is more Compton-like: the gravitons scatter off formion lines, via two $T_{\mu \nu}^{F}$ currents through virtual formion exchange (in direct as well as crossing versions). The schematic expression for the total amplitude should look like $M_{4}^{g g F F} \sim \kappa^{2} R^{2} F^{2}$ up to derivatives and the exchange pole. [There is no simple $\mathrm{D}=4$ reduction available here since a 4 -form is a constant in $\mathrm{D}=4$.] To perform the detailed calculations it proved useful to employ the program FORM [1흔.

As yet we can only give the amplitude in semi-final form, before the graviton polarizations have been converted into curvatures, but with the formions entirely expressed in terms of their field strengths. The eventual "FFRR" form is guaranteed by the (verified) invariance of $\mathrm{M}$ under graviton gauge transformations. The amplitude $M_{4}^{g g F F}$, before (stu) multiplication, reads

$$
\begin{aligned}
& \frac{1}{6 s}\left(F_{12}^{\mu_{1} \nu_{1}} \epsilon_{1 \mu_{1}} \epsilon_{2 \nu_{1}} \epsilon_{2} \cdot p_{2} \epsilon_{1} \cdot p_{1}-\right. \\
& 3 F_{12}^{\mu_{1} \mu_{2} \nu_{1} \nu_{2}} \epsilon_{2 \mu_{1}} \epsilon_{1 \mu_{2}} k_{2 \nu_{1}} \epsilon_{2 \nu_{2}} \epsilon_{1} \cdot p_{1}- \\
& 3 F_{12}^{\mu_{1} \mu_{2} \nu_{1} \nu_{2}} k_{1 \mu_{1}} \epsilon_{1 \mu_{2}} \epsilon_{1 \nu_{1}} \epsilon_{2 \nu_{2}} \epsilon_{2} \cdot p_{2}- \\
& 6 F_{12}^{\mu_{1} \mu_{2} \mu_{3} \nu_{1} \nu_{2} \nu_{3}} k_{1 \mu_{1}} \epsilon_{2 \mu_{2}} \epsilon_{1 \mu_{3}} k_{2 \nu_{1}} \epsilon_{1 \nu_{2}} \epsilon_{2 \nu_{3}}+ \\
& \left.3 F_{12}^{\mu_{1} \mu_{2} \nu_{1} \nu_{2}} k_{1 \mu_{1}} \epsilon_{1 \mu_{2}} k_{2 \nu_{1}} \epsilon_{2 \nu_{2}} \epsilon_{1} . \epsilon_{2}\right)+ \\
& +s \leftrightarrow \text { uperm. } \\
& \frac{1}{6 t} F_{12}^{\mu_{1} \nu_{1}}\left(\epsilon_{2 \mu_{1}} \epsilon_{1 \nu_{1}} \epsilon_{2} \cdot k_{1} \epsilon_{1} \cdot k_{2}+\epsilon_{1 \mu_{1}} \epsilon_{2 \nu_{1}} \epsilon_{2} \cdot k_{1} \epsilon_{1} \cdot k_{2}\right. \\
& -\frac{1}{2} k_{1 \mu_{1}} k_{2 \nu_{1}}\left(\epsilon_{1} . \epsilon_{2}\right)^{2}-\frac{1}{2} k_{2 \mu_{1}} k_{1 \nu_{1}}\left(\epsilon_{1} . \epsilon_{2}\right)^{2} \\
& \left.-\mathcal{F}_{1}^{\mu_{1} \alpha_{1}} \epsilon_{2 \alpha_{1}} \mathcal{F}_{1}^{\nu_{1} \alpha_{1}} \epsilon_{2 \alpha_{1}}-\mathcal{F}_{2}^{\mu_{1} \alpha_{1}} \epsilon_{1 \alpha_{1}} \mathcal{F}_{2}^{\nu_{1} \alpha_{1}} \epsilon_{1 \alpha_{1}}\right) \\
& +\frac{1}{12}\left(F_{12}^{\mu_{1} \nu_{1}} \epsilon_{2 \mu_{1}} \epsilon_{1 \nu_{1}} \epsilon_{2} . \epsilon_{1}+F_{12}^{\mu_{1} \nu_{1}} \epsilon_{1 \mu_{1}} \epsilon_{2 \nu_{1}} \epsilon_{1} . \epsilon_{2}\right. \\
& \left.+6 F_{12}^{\mu_{1} \mu_{2} \nu_{1} \nu_{2}} \epsilon_{1 \mu_{1}} \epsilon_{2 \mu_{2}} \epsilon_{1 \nu_{1}} \epsilon_{2 \nu_{2}}+\frac{1}{8} F_{12}\left(\epsilon_{1} . \epsilon_{2}\right)^{2}\right)(.23)
\end{aligned}
$$

The last, local, term includes the 4-point vertex as well as local contributions from the other graphs. The notation is as follows: $k_{i}, p_{i}$ denote respectively the graviton and formion momenta, $F_{12}^{\mu_{1}, \cdots \mu_{i} \nu_{1}, \cdots \nu_{i}}$ is the product of the field strengths of formions 1 and 2 , with the last $4-i$ indices contracted, while $\mathcal{F}_{i}^{\mu_{1} \mu_{2}}$ stands for the invariant combinations $k_{i}^{\mu_{1}} \epsilon_{i}^{\mu_{2}}-k_{1}^{\mu_{2}} \epsilon_{i}^{\mu_{1}}$. As in Sec. 2, the polarization tensor of each graviton is represented as the product of two polarization vectors, $\epsilon_{i}$. With these conventions, the amplitude is symmetric under $s-u$ interchange, corresponding to interchange of the (1-2) gravitons, while the $1 / t$ term is then separately invariant under (1-2).

In summary, the set of scattering amplitudes $(13,16,17,21,23)$ displayed here represents the bosonic part of the advertised linear 4-point SUSY invariant.

Above, we first constructed and then localized the (bosonic) four-point tree amplitudes to obtain the bosonic part of a linearized SUSY invariant quartic in the field strengths $(F, R)$. Here we discuss some consequences of this invariant's existence on the issue of renormalizability of $D=11$ SUGRA. In this connection a brief review of the general SUGRA divergence problem as it applies to $\mathrm{D}=11$ may be useful. For clarity, we work in the framework of dimensional regularization, in which only logarithmic divergences appear and consequently the local counterterm must have dimension zero (including dimensions of the coupling constants in the loop expansion); the generic gravitational loop expansion proceeds in powers of $\kappa^{2}$ (we will separately discuss the effect of the additional appearance of $\kappa$ in the CS vertex). It should also be stated (in connection with another $\kappa^{2}$ counting) that while the present discussion really proceeds at lowest order in an expression about flat space, with linearized curvatures, etc, the "covariantly dressed" quantities enter through including additional graviton lines at each graviton vertex; this will not alter the divergence countings, although it can be extremely complicated to achieve. Indeed, the same can be said of the whole process of reaching the fully locally SUSY invariant version of our 4-point amplitudes: it must exist just because it comes from the underlying action (1), as the physical expression of scattering among asymptotically defined states, though that does not make the perturbative resummation very obvious! 
At one loop (omitting the overall "infinite" $1 / \epsilon$ factor $)$, the counter-action would be $\triangle I_{1} \sim$ $\kappa^{0} \int d x^{11} \triangle L_{1}$. But there is no candidate $\triangle L_{1}$ of dimension 11, since odd dimension cannot be achieved by a purely gravitational $\triangle L_{1}$. ["Gravitational" $\sim \epsilon \Gamma R^{4}$ or "form-gravitational" $\sim \epsilon A R^{4}$ (respectively parity odd and even) CS-like terms [14i] cannot arise perturbatively i.e., with integer powers of $\kappa$.] Possible invariants involving odd powers of $\kappa$ arising from the CS vertex also cannot give rise to 1 -loop diagrams. These candidates, consisting of a polygon (triangle or higher) with form/graviton segments and appropriate emerging external bosons at its vertices, have as simplest example a form triangle with three external $F$-lines $\sim \kappa^{3} \int d^{11} x \partial^{9} \epsilon A F F$. However, this odd number of derivatives clearly cannot yield a scalar. The same counting also excludes the oneloop polygon's gravitational or form extensions such as $F^{2} R, F R^{2}$ or even $F^{3} R$ at this $\kappa^{3}$ level.

At two loops, $\triangle I_{2} \sim \kappa^{2} \int d^{11} x \triangle L_{2}$, so that $\triangle L_{2} \sim[L]^{-20}$ which can be achieved (to lowest relevant, $4^{\text {th }}$, order in external lines) by (for the pure graviton contribution) $\triangle L_{2} \sim \partial^{12} R^{4}$, where $\partial^{12}$ means twelve explicit derivatives spread among the 4 curvatures. There are no relevant 2 -point $\sim \partial^{16} R^{2}$ or 3 - point $\sim \partial^{14} R^{3}$ terms because the $R^{2}$ can be field-redefined away into the Einstein action in its leading part (to $h^{2}$ order, $E_{4}$ is a total divergence in any dimension) while $R^{3}$ cannot appear by SUSY. This latter fact was first demonstrated in $\mathrm{D}=4$ but must therefore also apply in higher $\mathrm{D}$ simply by a direct dimensional reduction argument. Thus the terms we need are, for their 4-graviton part, $L_{4}^{g}$ of (13) with twelve explicit derivatives. The companions of $L_{4}^{g}$ in $L_{4}^{\text {tot }}$ will simply appear with the same number of derivatives. It is easy to see that the additional $\partial^{12}$ can be inserted without spoiling $S U S Y$; indeed they appear as naturally as did multiplication by stu in localizing the $M_{4}$ to $L_{4}$ : for example, $\partial^{12}$ might become, in momentum space language, a combination of $\left(s^{6}+t^{6}+u^{6}\right)$ and $(s t u)^{2}$, spread according to rules similar to those presented in the text. This establishes the structure of the 4-point local counterterm candidate we are considering.

Before the present construction of the complete counterterm was completed, the actual co- efficient of its 4-graviton part was computed $\left[\begin{array}{l}4 \\ 1\end{array}\right]$ by a combination of string-inspired and unitarity techniques. The structure of infinities in the fourgraviton sector for all maximal supergravities up to two loops was extensively studied there, and conjectures on higher loops were presented as well. [Very recently, a parallel analysis of type I supergravities has been carried out in [1] for completeness we state the methods and relevant final results of [iי4]: Begin by computing the tree supergravity amplitudes by means of the KLT relations. Next, use these tree amplitudes as input for the cutting rules to obtain the analytic structure of the one-loop amplitudes at any $D$. This information, because of the high degree of supersymmetry, is enough to reconstruct the one-loop amplitudes. Now iterate the procedure and go to two loops. [What makes the procedure quite cumbersome beyond two loops is the increasing number of $n$-particles cuts that one has to examine to reconstruct the amplitude .] Finally compute the eventual divergences; in $D=11$ as we saw on general ground there is no 4-point one-loop divergence, while at two loops the calculation yields the explicit infinite result

$$
\begin{gathered}
\left.\triangle I_{2}(g)\right|_{\text {pole }}=\left(\frac{\kappa}{2}\right)^{6} \frac{1}{48 \epsilon(4 \pi)^{11}} \frac{\pi}{5791500} \times \\
\left(438\left(s^{6}+t^{6}+u^{6}\right)-53 s^{2} t^{2} u^{2}\right)\left(s t u M_{4}^{\text {g tree }}\right)(24)
\end{gathered}
$$

for the local 4-graviton divergence, dimensionally regularized to $\mathrm{D}=11-2 \epsilon$, with $(s t u) M_{4}^{\mathrm{g}}$ tree $\equiv L^{g}$ of (11). The results discussed previously then embody the extension of $\left(2_{4}^{\prime}\right)$ to the complete bosonic sector counterterm.

We have succeeded in constructing explicitly the tree level nonlocal 4-point scattering amplitudes involving the two bosons of $\mathrm{D}=11$ SUGRA, namely the graviton and formion, as well as obtaining the corresponding local invariant in a SUSYpreserving way. Extending the result to the rest of the amplitude, involving two or four gravitinos, is not that difficult in terms of the techniques employed here [3]: the gravitino primarily interacts with the graviton through its stress tensor $\sim k^{\mu \nu} T_{\mu \nu}(\psi)$, and with the formion through a simple (nonminimal) coupling term $\sim(\bar{\psi} \Gamma \psi F)$. The (complicated) 4-fermion contact terms are needed, but only for the 4-fermion part of the 
amplitude, where they insure the SUSY invariance, not for the 2-gravitino to 2-boson amplitudes. In any case the bosonic part alone, if SUSY-transformed, will provide a complete linearized SUSY invariant. In addition to its intrinsic interest as a example of a "physical" process in $\mathrm{D}=11$ SUGRA, the result was of primary interest to us as confirmation of the existence of an invariant that, (in its localized version) has the dimension of a candidate counterterm for (dimensionally regularized) 2-loop infinities. Indeed, its 4-graviton part agreed completely with the coefficient of the 2-loop infinity recently calculated in that sector in [4] , while its 4 -formion part agreed with a very different matrix-theory motivated scattering calculation [1 $\left.12^{\prime}\right]$. The existence of infinities in this ultimate local SUGRA model, while not unexpected from a purely power counting field theoretical point of view, is important in showing that no hidden symmetry rescues this most unique theory. Of course such a putative symmetry could still suppress all higher loop infinities beyond a certain order, but this seems

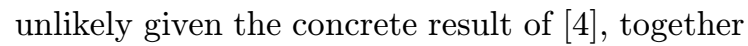
with the obvious constructibility of higher order candidate counterterms e.g., using the scattering approach. We can at least conclude that the case for underlying finite extended (M-)theories is thereby strengthened. In this connection, we emphasize that the invariant found here has a further interest as another example (see also [1 $1 \overline{6}_{1}^{\prime \prime}$, [1] $\left.\overline{7}_{1}\right]$ ) of possible local corrections to M-theory whose leading term is presumably the action (1). This might teach us something about this underlying model, just as the corrections to the Einstein action in slope expansion of the various $\mathrm{D}=10 \mathrm{su}-$ perstring models could be understood from the latters' properties; persistence in $\mathrm{D}=11$ of the " $t_{8} t_{8}$ " $\mathrm{D}=10$ string theory hallmark is perhaps one first hint about the M-string connection.

\section{Acknowledgments}

I am deeply indebted to my $\mathrm{D}=11$ coauthor $\mathrm{S}$. Deser for many discussions on this subject. I am also grateful to especially to Z. Bern, L. Dixon, and D. Dunbar for discussions of their work and mine, as well as to A. Waldron, who also introduced me to the world of FORM. This work was supported by the National Science Foundation under grant PHY99-73935.

\section{References}

[1] E. Cremmer, B. Julia and J. Scherk, Phys. Lett. B 79, 409 (1978).

[2] S. Deser, "Infinities in quantum gravities", grqc/9911073.

[3] S. Deser, D. Seminara, A. Waldron (in progress).

[4] Z. Bern, L. Dixon, D.C. Dunbar, M. Perelstein, J.S. Rozowsky, Nucl. Phys. B530, 401 (1998).

[5] S. Deser and D. Seminara, Phys. Rev. Lett. 82, 2435 (1999).

[6] S. Deser, J. S. Franklin and D. Seminara, Class. Quant. Grav. 16, 2815 (1999).

[7] S. M. Christensen, S. Deser, M. J. Duff and M. T. Grisaru, Phys. Lett. 84B, 411 (1979).

[8] H. Kawai, D. C. Lewellen and S. H. Tye, Nucl. Phys. B269, 1 (1986).

[9] J.H. Schwarz, Phys. Rept. 89, 223 (1982).

[10] S. Sannan, Phys. Rev. D 35, 1385 (1987).

[11] S. Deser and J. H. Kay, Phys. Lett. B76, 400 (1978).

[12] J. Plefka, M. Serone and A. Waldron, JHEP 9811, 010 (1998).

[13] J.A.M Vermaseren,Symbolic Manipulation with FORM, version 2, (Computer Algebra Nederland, Amsterdam, 1991).

[14] L. Alvarez-Gaume and E. Witten, Nucl. Phys. 234B, 269 (1984); M.J. Duff, J.T. Liu and R. Minesian, Nucl. Phys. B452, 261 (1995).

[15] D. Dunbar, B. Julia, D. Seminara and M. Trigiante, "Counterterms in type I supergravities", hep-th/9911158.

[16] M.B. Green, M. Gutperle and P. Vanhove, Phys. Lett. B 409, 177 (1997); M. B. Green, H. Kwon and P. Vanhove, "Two loops in eleven dimensions", hep-th/9910055; M. B. Green and P. Vanhove, "The low energy expansion of the one-loop type II superstring amplitude", hepth/9910056.

[17] J.G. Russo and A.A. Tseytlin, Nucl. Phys. B 508, 245 (1997). 